\title{
医学图像数据压缩中的机器学习方法
}

\author{
侯宇轩，邸奕宁，任重，陶显波，陈为 \\ (浙江大学 CAD\&CG 国家重点实验室 杭州 310058) \\ (renzhong@cad.zju.edu.cn)
}

\begin{abstract}
摘 要: 在医学研究中会产生大量需要储存、传输的图像数据, 如计算机断层扫描 $(\mathrm{CT})$ 等. 医院要处理海量患者的所 有数据相当困难, 必须对这些数据进行压缩. 随着人工智能技术的发展, 应用机器学习的医学图像数据压缩方法成 为新的研究热点. 文中首先总结了传统医学图像数据压缩方法; 然后基于机器学习的医学图像数据压缩方法, 对各 类方法针对脑部 $\mathrm{CT}$ 、肺部 $\mathrm{CT}$ 等不同医学图像的压缩效果进行对比, 并对这些方法在压缩率、算法复杂度、重构质 量等方面的优缺点进行系统的总结, 指出机器学习和感兴趣区域结合的方法可以在享受有损压缩带来的高压缩率的 同时保留重要区域的特征信息, 比其他压缩方法更贴合医学数据的压缩要求; 最后展望了该领域的未来方向和挑战.
\end{abstract}

关键词: 医学图像; 图像压缩; 机器学习

中图法分类号: TP391.41 DOI: 10.3724/SP.J.1089.2021.18687

\section{Machine Learning Methods in Medical Image Compression}

\author{
Hou Yuxuan, Di Yining, Ren Zhong", Tao Yubo, and Chen Wei
}

(State Key Laboratory of CAD\&CG, Zhejiang University, Hangzhou 310058)

\begin{abstract}
A large amount of image data such as CT that needs storage and transmission is generated in medical research. It is hard for the hospital to handle all data of the numerous patients. Therefore, it is of vital importance to compress these image data. Recently, learning-based medical image compression has become a new research trend with the development of artificial intelligence. Traditional methods in medical data compression are firstly reviewed. Further study in learning-based approaches is made, and the compression performance of these approaches in different medical image data such as brain CT and liver CT are shown. In the meantime, the advantages and disadvantages of these approaches in various aspects such as compression ratio, algorithm complexity and reconstruction quality are systematically summarized. It is pointed out that the combination of learning-based method and ROI-based method achieves high compression ratio brought by lossy compression, while keeping the feature information of the critical regions. Consequently, this approach is much more suitable for medical image compression than others. Finally, the paper concluded with a discussion of future development in this field.
\end{abstract}

Key words: medical image; image compression; machine learning

收稿日期：2020-09-29; 修回日期：2020-11-16. 基金项目：国家重点研发计划(2019YFB1404802); 国家自然科学基金 (61890954). 侯宇轩(1996-), 男, 博士研究生, 主要研究方向为医学图像处理; 邸奕宁(1998一), 男, 在校学生; 任重(1978一), 男, 博士, 副教授, 博士生导师, 论文通讯作者, 主要研究方向为实时绘制、虚拟现实; 陶暗波(198一), 男, 博士, 副教授, CCF 会员, 主 要研究方向为数据可视化、可视分析; 陈为(1976-), 男, 博士, 教授, 博士生导师, CCF 杰出会员, 主要研究方向为可视化、可视分 析、大数据分析、人机混合智能. 
随着医学成像技术的发展和仪器扫描精度的 提高, 扫描产生的医学图像数据量越来越大. 以常 用的计算机断层扫描(computer tomography, CT)为 例, 每幅 $\mathrm{CT}$ 图像是由数张到数百张切片组成的, 占用空间可达数百兆字节. 此外, 在临床中经常要 求患者的诊疗影像数据保存至少数月. 数据的采 集量、存储需求量的增加, 要求存储医学图像数据 时使用图像压缩技术(或称为图像编码技术). 此 外, 现代医学中的远程医疗技术, 使来自偏远乡村 的患者也能从城市中有经验的医生那里得到咨询. 在远程医疗中, 由于网络带宽的限制, 同样必须对 医学图像进行压缩后再传输. 因此, 医学图像压缩 已经成为诊疗中不可或缺的部分.

现有的压缩技术分为有损压缩和无损压缩. 其中, 无损压缩是可逆的, 数据不会因压缩而产生 任何损失; 有损压缩是不可逆的, 解压后的图像与 原图会有所差别. 然而, 有损压缩可以达到非常高 的压缩比率，这是无损压缩所无法企及的.

在多媒体领域，有损压缩的结果一般只要视 觉上无损就可以被接受; 而医学图像领域则要求 更严格, 因为临床数据的丢失可能会损害影像检 查对患者的价值. 起初, 医学界普遍要求使用无损 压缩, 但随着数据体量的增加, 仅仅使用无损压缩 无法满足存储和传输需求; 之后, 美国食品药品监 督管理局宣称，可以使用有损压缩，但在显示压缩 图像时必须标明该图像是有损的 ${ }^{[1]}$. 在保证诊断可 接受性的前提下，采用有损压缩会使图像的存储 负担大大减少. 如果选择在工作站上对图像进行 解压缩, 网络带宽的需求同时也将减少.

\section{1 传统压缩方法}

应用于医学图像压缩中的有损压缩技术分为 基于向量量化(vector quantization, VQ)的方法、基 于变换编码的方法和基于分形压缩的方法，它们 都能达到较高的压缩比. 其中，基于变换编码的方 法属于对称压缩方法，压缩和解压的时间相差不 大；而 VQ方法和基于分形压缩的方法属于非对称 压缩方法, 压缩需要耗费大量的搜索时间, 解压则 相当高效. 根据它们的特性, 长期存储适合使用非 对称压缩方法, 而实时远程医疗适合使用对称压 缩方法.

应用于医学图像压缩中的无损压缩技术有熵 编码(如 Shannon 编码、Huffman 编码和算术编码)、 游程编码等方法, 用于对有损压缩后的结果再编
码, 进一步提高压缩比例. 如果单独使用无损编 码, 压缩比例一般为 $1: 8$.

\section{$1.1 V Q$ 方法}

VQ 方法将要压缩的变换图像分解成向量, 将 这些向量分割成聚类, 这些聚类的中心称为码字, 所有码字的集合称为码本. 产生码本之后, 将各个 向量量化到最接近的码字, 则每个向量都可以通 过它对应的码字的二进制地址来表示. 该方法利 用图像块之间的相关性和圥余性实现压缩，被广 泛应用于图像编码中. 基于 VQ 的压缩得到的压缩 比一般为 $1: 8 \sim 1: 20$.

尽管使用 VQ 的压缩相对无损压缩可以提供 更高的压缩比，但其使用搜索生成码本的时间较 长, 码本存储也需要额外空间. 另外, VQ 编码容 易出现边缘退化问题.

\section{2 变换编码方法}

基于变换编码的压缩利用不同的变换方法, 将图像从像素域变换到其他域(一般是频域), 再将 变换后得到的系数进行量化等操作完成压缩. 变 换包括离散余弦变换(discrete cosine transform, DCT) 和离散小波变换(discrete wavelet transform, DWT)等. 其中, 基于 DWT 的方法一般比基于其 他变换的方法得到更好的图像质量或更高的压缩 比. 许多方法作为图像压缩的标准, 如联合图像专 家组(joint photographic experts group, JPEG) ${ }^{[2]}$ 、嵌人 式零树小波 (embedded zerotree wavelets, EZW) ${ }^{[3]}$ 和 多级树集合分裂 (set partitioning in hierarchical trees, SPIHT) ${ }^{[4]}$, 都使用 DWT. 它们的压缩率很高, 但是随着压缩率的增加, 在对分块后的图像进行 压缩时会由于量化步长过大而丢失大量边缘高频 信息, 产生块效应, 即解码后分块边缘产生不连续 现象. 基于变换编码压缩的压缩比一般为 1:8 1:20.

\section{3 分形压缩方法}

分形压缩方法是一种特殊的压缩方法. 其基 本思想是借鉴自然界中的雪花分形等自相似图案, 利用图像中的拓扑自相似性将图像的各个区域通 过适当的变换相互表达. 解码时, 通过分形函数迭 代处理恢复生成解码后的图像. 该方法对具有良 好的正则性和自相似性的图像压缩非常有效, 压 缩比甚至能超过 100 倍. 但是, 分形压缩要求每个 小块图像都在整个空间中搜索与自己相似度最大 的、分辨率更低的大块图像, 有非常多的重复计算, 因此编码相当慢.

\section{4 感兴趣区域/感兴趣体积技术}

通常, 医学图像同时包含人体的组织区域和 
背景区域，信息十分丰富. 其中，一些区域对诊断 至关重要, 如肿瘤区、病变区或脑活动区，放射科 医生可能无法容忍高压缩比下这些区域的信息损 失.一种对重要诊断区域不压缩或进行无损压缩、 而其他背景区域应用有损压缩的技术称为感兴趣 区域/感兴趣体积(region of interest/volume of interest，ROI/VOI)压缩技术，需要无损压缩的区域 称为 ROI/VOI(下文统一以 ROI 代替). 这样的压缩 可以在大幅减小图像存储大小的同时保留重要的 临床诊断信息，在医学图像处理中有很大的潜力. 但是，传统的分割 ROI 的技术基于区域生长法、活 动边界法或边缘检测法, 精度较低, 耗时长, 同时
经常需要额外的人工干预，导致 ROI 技术难以投 人实际应用.

\section{5 其他传统方法}

根据三维体数据和视频数据在结构上的相似 性, 将视频压缩方法用于体数据压缩非常有潜力. 高效视频编码(high efficiency video coding, HEVC) 是最先进的视频压缩方法, 能在相同压缩质量下 比其前代 H.264 节省一半空间. 虽然该方法需要大 量复杂的计算, 但是 GPU 的广泛使用使得其计算 效率大大提高. 目前, HEVC 和 JPEG2000 一样被 写人医学图像传输标准. 传统医学图像压缩方法 统计如表 1 所示.

表 1 传统医学图像压缩方法统计

\begin{tabular}{llll}
\hline 方法分类 & \multicolumn{1}{c}{ 原理 } & \multicolumn{1}{c}{ 优势 } \\
\hline $\mathrm{VQ}$ & 将图像量化到预设好的向量(码字) & 压缩率较高, 解码速度快 & $\begin{array}{l}\text { 生成码本搜索时间长, 编码时间 } \\
\text { 势, 对初始码本选择敏感 }\end{array}$ \\
& & & \\
变换编码 & 将图像变换到频域, 利用频域的 & 压缩率较高, 一般使用的变换有较为成熟的 & 随压缩率的提高, 产生块效应 \\
& 罙余特征(如图像高频部分) & 方法, 编码速度快 & \\
分形 & 利用图像的自相似性 & 压缩率极高, 对有自相似性的图像效果非常 & 计算复杂, 编码慢 \\
& & 好, 解码快, 解码速度与图像分辨率无关 & \\
\hline
\end{tabular}

\section{2 基于机器学习的医学图像压缩}

随着机器学习的发展, 医学图像压缩和机器 学习产生了很多成功的结合方案.

\section{1 机器学习结合 VQ 方法}

$\mathrm{VQ}$ 将图像分解为向量, 并将这些向量量化到 距离它们最近的码字. 这些码字的集合称为码本, 一般是通过聚类方法生成的. 之后，使用这些码字 在码本中的位置就可以重构原始图像.

$K$-means 聚类方法常用于 VQ 的码本生成，该 方法通过不断计算点到各聚类的平均距离修正聚 类中心. 如 Setiawan 等 ${ }^{[5]}$ 应用 $K$-means 方法探索彩 色眼底图像在不同彩色通道下使用 VQ 压缩的效 果. 然而, $K$-means 方法存在容易陷人局部极小值、 结果对初始码本敏感等问题.

为了解决传统 VQ 方法的边缘退化问题, Jiang 等 ${ }^{[6]}$ 将图像分割为不同分辨率的块，低分辨率的块 使用无损的 Huffman 编码压缩，高分辨率的块使 用 VQ 进行压缩; 同时，借鉴分形压缩中分形维度 的概念，通过计算块的分形维度来选取初始码本， 使依赖初始码本的 $K$-means 方法效果更好，在肝脏 $\mathrm{CT}$ 图像和脑部 $\mathrm{CT}$ 图像上取得了良好的效果.

Kouanou 等 ${ }^{[7]}$ 使用 $K$-means 方法结合分裂方法 加速 VQ 的码字计算. 若一个聚类过大，就将其分 裂为 2 个聚类, 使用 2 个新的码字代替原有码字,
在一定程度上解决了聚类不平衡的问题，更容易 得到全局最优值. 该方法在一般 X 射线图像、乳腺 钿靶图像、腹部 CT 图像等多种图像上进行测试, 获得的重构图像质量较高.

$\mathrm{Hsu}^{[8]}$ 首先使用分水岭方法分割出肿瘤，将图 像分为肿瘤区、非肿瘤前景区和背景区 3 部分; 之 后将竞争神经网络 (competitive Hopfield neural network, CHNN)引人 VQ 中，对不同的区域进行不 同级别的量化, 以同时保留重要的肿瘤特征, 减小 乳腺图像的大小, 提高存储效率. 实验结果表明, 该方法在乳腺锄靶图像的压缩应用中取得良好的 效果，并且减少了原本基于小波的 VQ 方法带来的 图像损失. 但是, 分水岭方法受初始化影响大, 容 易产生过分割，对后续压缩产生影响.

Meyer-Bäse 等 ${ }^{[9]}$ 提出一种基于保拓扑神经网 络的 VQ 编码方法, 用于乳腺钿靶图像的压缩. 该 方法改进基于 EZW 方法的 VQ 方法，可以应用于 较大的或重叠的分块, 减小了重构后图像的块效 应. 向量量化过程由一个称为神经气体网络的保 拓扑神经网络执行, 该网络可以快速收玫, 并且比 传统搜索码本的 $K$-means 方法误差更小, 对初始化 码本的敏感性也有所降低.

Ashraf 等 ${ }^{[10]}$ 提出一种采用 VQ 和自组织特征 映射网络(self-organizing feature mapping, SOFM) 结合的医学图像压缩技术, 并在文献[11]中分析了 
其表现. SOFM 由 Kohonen ${ }^{[12]}$ 提出, 也是一种保拓 扑的神经网络, 广泛应用于医学图像处理中. 与 $\mathrm{CHNN}^{[8]}$ 中只有竞争获胜的神经元才能调整权值 不同, 使用 SOFM 的聚类是一种模糊式的聚类方 法, 每个向量不仅影响与它距离最近的聚类, 同时 也对全局的其他所有聚类有所调整, 可以实现码 本的自适应选择. 该方法是一种混合有损-无损压 缩方法，首先使用有损方法(VQ)压缩，之后将重 构结果和原始图像的差异使用无损的 Huffman 编 码压缩, 再一并进行传输, 重构可以得到无损的图 像. 在肺部 $\mathrm{X}$ 射线图像、脑部核磁共振 (magnetic resonance, MR)图像、肝脏 $\mathrm{CT}$ 图像等多种数据的 测试结果显示, 该方法比传统的无损方法获得了 更高的压缩率.

Guo 等 ${ }^{[13]}$ 同样使用 SOFM 实现 VQ 压缩，之后 又使用无损的差分编码对 VQ 的压缩结果进行二 次压缩. SOFM 的保拓扑性质使得原本连续的块在 经过神经网络输出后仍然是相似的, 使得差分编 码的压缩率大大提高.

赵明等 ${ }^{[14]}$ 则针对 SOFM 的 2 个不足, 即缺乏 数据分布的信息导致数据中心难以估计，使用不 充分的问题改进初始化后码本，使用动态方法调 整网络权重. 该方法提升了编码效率，并改善了医 学图像的重建质量.

除了使用神经网络提高 VQ 的码本质量外, $V Q$ 和机器学习方法也可以直接组合. Karl $\mathrm{k}^{[15]}$ 将 $\mathrm{VQ}$ 和神经网络结合压缩脑部 $M R$ 图像, 将 $V Q$ 的 输出作为神经网络的输人进行二次压缩, 得到比 单纯使用 VQ 压缩更高的压缩率.

\section{2 机器学习结合变换编码类方法}

Northan 等 ${ }^{[16]}$ 将神经网络看做一个滤波器组, 对高频信号和低频信号分别处理，代替 SPIHT 原 本的滤波器, 得到一个在脑部 MR 图像上泛化性能 非常好的压缩网络.

Khashman 等 ${ }^{[17]}$ 未让神经网络直接参与压缩, 而是针对使用 Haar 小波的压缩作参数预测. 对于 每个要压缩的数据, 神经网络会在压缩率和图像 质量之间做出权衡，给出使用 Haar 小波变换压缩 该图像的最优参数. 但是, 在一般 $\mathrm{X}$ 射线图像上的 测试结果表明，该方法的压缩质量不高，难以在实 际医学场景中应用.

Vijideva ${ }^{[18]}$ 将神经网络作为 EZW 方法的预处 理方法，向网络中输人减去背景的图像试图重构 原始图像, 进行重复训练, 直到网络重构图像和原 始图像的差距足够小; 再使用 EZW 方法和游程编
码进行压缩. 在肌肉 MR 图像上的测试结果表明, 该方法的压缩质量较高, 损失很小.

脊波变换是一种新的多尺度变换方法，比小 波变换提取图像特征的能力更强. Saudagar 等 ${ }^{[19]}$ 将 图像分割为不同尺度的块, 并分别应用有限脊波 变换, 变换之后的结果输人一个组合神经网络进 行压缩, 代替传统变换编码的量化步骤. 在一般 MR 图像上的测试结果表明, 该方法比传统的 JPEG2000 方法压缩耗时更少, 但是图像质量有所 下降.

\section{3 机器学习结合分形类方法}

分形编码技术比较耗时，尤其是其中的搜索 匹配过程有大量的穴余计算. Bhavani 等 ${ }^{[20]}$ 通过 SOFM 将相似的搜索空间直接经过映射集中在一 起, 大大加快了分形编码的搜索速度, 在脑部 MR 图像的压缩测试中不仅保留了一般分形压缩方法 的质量，还将压缩时间缩短到原来的约 $1 / 50$.

Lakshmi 等 ${ }^{[21]}$ 使用神经网络训练一个专家系 统，用于减少分形压缩中的搜索空间大小，该专家 系统会针对需要匹配的区域, 从所有备选区域中 自动推荐与其相似性较高的若干区域，降低搜索 复杂度. 该方法在脑部 MR 图像压缩中比一般分形 压缩方法在质量和速度上都有小幅提升.

Perumal 等 ${ }^{[22]}$ 未采用加速搜索的方法, 而是在 分形压缩之后使用径向基神经网络(neural network with radio basis function, NNRBF)进行二次压缩. 在脑部 CT 图像、脑部 MR 图像的测试结果表明, 该 方法得到了更高的压缩率，但由于 2 个阶段均为有 损压缩方法，该方法重构图像的误差较大.

\section{4 机器学习用于分割 ROI 的方法}

对基于 ROI 的方法，如果每次都需要人工标 注 ROI, 那么该压缩方法对于用户是不友好的. 但 是, 利用机器学习方法解决医学图像的分割问题 目前已取得大量的研究成果. Thomas 等 ${ }^{[23]}$ 使用 $K$-means 聚类得到 ROI 部分, 对这部分应用无损的 快速曲波变换和算术编码压缩，其余部分则使用基 于小波变换的 VQ 压缩，得到一种快速且高质量的、 用于压缩脑部 MR 图像的混合有损-无损编码方法.

随着神经网络在医学图像分割领域的发展, 出现了利用神经网络直接分割 ROI 用于压缩的方 法. Fan ${ }^{[24]}$ 使用 3D U-Net 作为 ROI 提取器, 之后结 合 SPIHT 方法进行编码. U-Net 是最先进的分割方 法之一, 而 SPIHT 是最先进的图像压缩方法之一, 该组合方法在三维脊椎 MR 图像上达到了较高的 压缩率和重构图像质量. 然而, 三维神经网络的训 
练对计算资源的要求很高, 这是一般医疗机构无 法达到的.

\section{5 直接使用机器学习方法进行压缩}

不是作为传统方法的辅助，而是直接利用机 器学习方法的压缩也是当前的研究热点之一. 由 于神经网络可以对任意映射进行拟合，一些学者 尝试直接使用神经网络压缩图像, 但是由于网络 参数搜索空间很大，得到的模型难以收玫. Durai 等 ${ }^{[25]}$ 通过估计一个累计分布函数将原始图像映射 到概率空间，从而加快神经网络的收玫速度; 然而 在腹部 MR 图像上的测试结果显示, 该方法的图像 重构质量较低. 魏欢欢 ${ }^{[26]}$ 利用提升小波方法将原始 图像进行预处理，使相邻像素幅值变化更小; 随后 使用多层前馈网络进行压缩, 在胸部 CT, MR 图像 压缩上获得了超过 EZW 方法的图像质量. Yeo 等 ${ }^{[27]}$ 选择减小模型容量, 直接使用如图 1 所示的 5 层前 馈神经网络对一般 MR 图像进行压缩, 得到的压缩 比很高, 但是压缩质量较低.

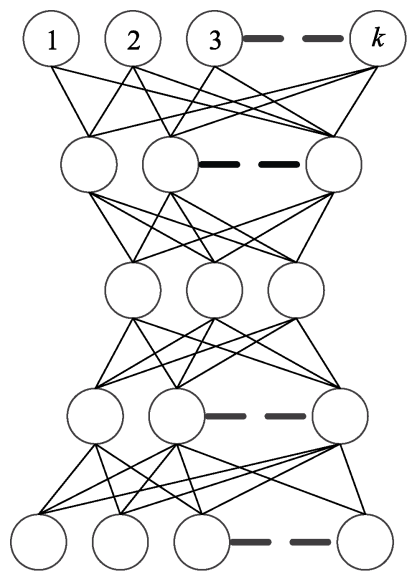

图 1 前馈神经网络 ${ }^{[27]}$

张诣 ${ }^{[28]}$ 将混沌映射与神经网络结合, 构造 2 种容错能力较强的混沌前馈神经网络用于压缩医 学图像, 并与一般全连接神经网络在脑部 MR 图像 和腹部超声图像上进行对比实验. 实验结果表明, 两者在重建图像的性能上比一般全连接神经网络 更优.

自编码器是神经网络的一种, 常用于各种数 据的降维操作. 很多学者未让神经网络直接输出 压缩后的图像，而是选择将原始数据输人自编码 器. 由于自编码器中间的隐藏层神经元数量小于 输人层的神经元数量, 因此隐藏层数据可以作为 原始数据的一个低维表示. 同时, 自编码器的输出 层和输人层大小相同, 输出层数据即为重构后的 图像. Tan 等 ${ }^{[29]}$ 利用受限玻尔兹曼机 (restricted
Boltzmann machine, RBM)压缩乳腺钿靶图像, 该 方法堆叠多层编码器, 为压缩提供多分辨率的选 择，如图 2 所示.

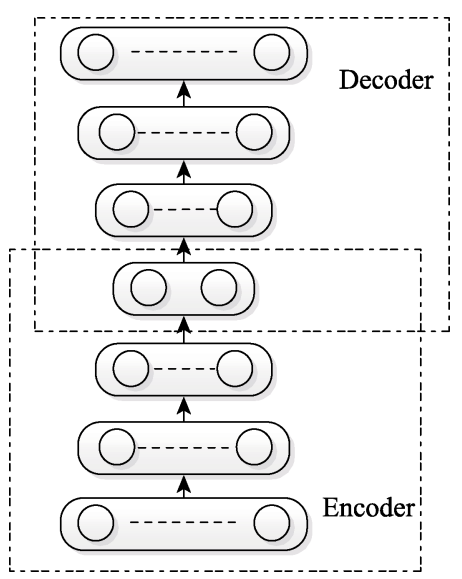

图 2 对自编码器的堆叠 ${ }^{[29]}$

Senthilkumaran 等 $^{[30]}$ 使用类似游程编码的方 法处理原始图像, 再将处理得到的向量输人自编 码器压缩. 该方法在一般 $\mathrm{X}$ 射线图像上的压缩质 量超过传统的 JPEG 方法, 与无损的 Huffman 编码 质量相近. Kar 等 ${ }^{[31]}$ 使用如图 3 所示新型的全卷积 自编码器进行压缩乳腺钿靶图像, 与传统自编码 器相比对图像边缘信息的提取能力更强, 且收玫 速度更快。该方法的重构图像质量超过了 JPEG, 但是没有超过 JPEG2000.
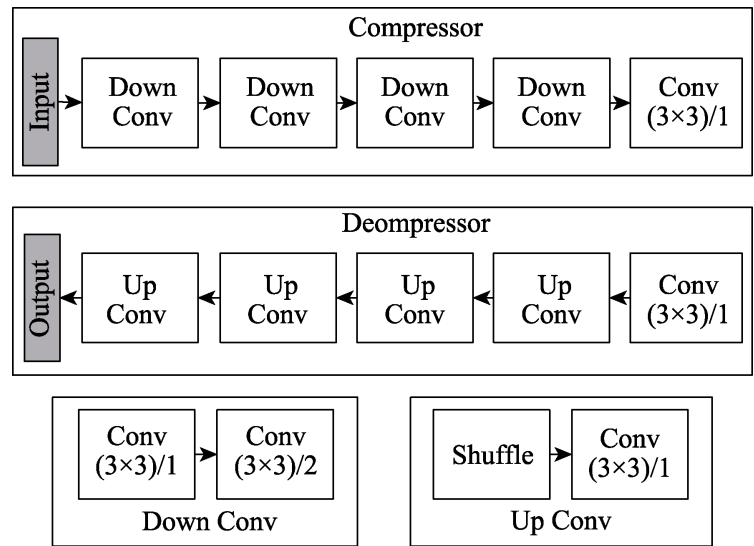

图 3 全卷积自编码器 ${ }^{[31]}$

Sushmit 等 ${ }^{[32]}$ 未使用自编码器, 而是采用最新 的循环卷积神经网络 (convolutional recurrent neural network, CRNN)压缩一般 X 射线图像. 该模型使 用多尺度方法, 可以提供可变的压缩率, 循环神经 网络又给该模型学习上下文有关特征带来了便利. 测试结果表明，该模型比 JPEG2000 模型得到的重 构质量更高. 上述模型的特性统计如表 2 所示. 
表 2 基于机器学习的医学图像压缩方法特性统计

\begin{tabular}{|c|c|c|c|c|c|}
\hline 方法分类 & 文献 & 出版年 & 特性 & 优势 & 劣势 \\
\hline \multirow{10}{*}{$\begin{array}{l}\text { 机器学习结 } \\
\text { 合 VQ }\end{array}$} & [5] & 2009 & $\begin{array}{l}\text { 使用 } K \text {-means 加速传统 VQ 寻找码本 } \\
\text { 的 LBG (Linde-Buzo-Gray)方法 }\end{array}$ & 比 LBG 方法更快 & $\begin{array}{l}\text { 容易陷人局部极小值, 对 } \\
\text { 初始码本敏感 }\end{array}$ \\
\hline & [6] & 2012 & $\begin{array}{l}\text { 借鉴分形维度概念生成初始码本供 } \\
K \text {-means 使用 }\end{array}$ & 减少了严重的边缘退化问题 & 预处理时间长 \\
\hline & [7] & 2018 & 使用 $K$-means 与分裂方法 & $\begin{array}{l}\text { 分裂方法减少 } K \text {-means 聚类不均衡的 } \\
\text { 问题 }\end{array}$ & $\begin{array}{l}\text { 分裂方法容易导致聚类 } \\
\text { 分割过细 }\end{array}$ \\
\hline & [8] & 2012 & $\begin{array}{l}\text { 使用分水岭方法和 CHNN 加速 VQ 的 } \\
\text { 学习过程 }\end{array}$ & 加快编码速度 & $\begin{array}{l}\text { 分水岭方法容易产生过 } \\
\text { 分割 }\end{array}$ \\
\hline & [9] & 2005 & 使用基于保拓扑映射的神经网络代替 & 与 $K$-means 相比, 不容易陷人局部极小 & VQ 码本选择的可解释性 \\
\hline & {$[10]$} & 2006 & VQ 的学习过程 & 值, 对初始化码本的敏感性降低 & 下降 \\
\hline & {$[11]$} & 2006 & & & \\
\hline & {$[13]$} & 2010 & & & \\
\hline & [14] & 2009 & & & \\
\hline & {$[15]$} & 2006 & $\begin{array}{l}\text { 将 VQ 的结果输人神经网络进行二次 } \\
\text { 压缩 }\end{array}$ & 提高压缩率 & 重构图像质量不高 \\
\hline \multirow{5}{*}{$\begin{array}{l}\text { 机器学习结 } \\
\text { 合变换编码 }\end{array}$} & {$[16]$} & 2006 & $\begin{array}{l}\text { 和 SPIHT 结合, 用于替代 SPIHT 里面 } \\
\text { 的滤波器组 }\end{array}$ & 泛化性能较好 & 训练需要时间长 \\
\hline & {$[17]$} & 2009 & 神经网络用于计算基于 Haar 小波的压 & 可以自动确定压缩超参数 & 受训练图像影响大, 泛化 \\
\hline & & & $\begin{array}{l}\text { 缩在压缩质量和压缩率平衡下的最优 } \\
\text { 配置参数 }\end{array}$ & & 能力不强, 压缩质量不足 \\
\hline & {$[18]$} & 2012 & 使用神经网络作为 EZW 的预处理 & 预处理精度高, 压缩图像质量提高 & 训练需要时间长 \\
\hline & {$[19]$} & 2014 & 脊波变换和神经网络结合 & $\begin{array}{l}\text { 使用神经网络代替传统量化过程, 不需 } \\
\text { 要图像的先验信息, 编码速度加快 }\end{array}$ & 图像质量下降 \\
\hline \multirow{3}{*}{$\begin{array}{l}\text { 机器学习结 } \\
\text { 合分形 }\end{array}$} & {$[20]$} & 2013 & 利用 SOFM 加速分形编码的搜索 & 大大加快编码速度 & 压缩质量受分形压缩质 \\
\hline & {$[21]$} & 2013 & $\begin{array}{l}\text { 利用神经网络训练专家系统减小搜索 } \\
\text { 区域加快分形编码搜索 }\end{array}$ & & 量限制 \\
\hline & {$[22]$} & 2016 & $\begin{array}{l}\text { 将分形压缩后的结果输人 NNRBF 进 } \\
\text { 行二次压缩 }\end{array}$ & 压缩率高 & 重构图像质量低 \\
\hline \multirow{3}{*}{$\begin{array}{l}\text { 机器学习结 } \\
\text { 合基于 ROI/ } \\
\text { VOI }\end{array}$} & {$[23]$} & 2014 & $\begin{array}{l}\text { 利用 } K \text {-means 等聚类方法用于 } \\
\text { ROI/VOI 的分割 }\end{array}$ & $\begin{array}{l}\text { 比人工勾画 ROI 或传统边缘检测法运 } \\
\text { 行快 }\end{array}$ & $\begin{array}{l}\text { 聚类方法需要平滑等后 } \\
\text { 处理 }\end{array}$ \\
\hline & [24] & 2018 & 利用 3D U-Net 用于 ROI 的分割 & U-Net 是最先进的医学图像分割方法之 & $\begin{array}{l}\text { 处理 } \\
\text { 需对图像分块压缩, 块的 }\end{array}$ \\
\hline & & & & 一, 分割 ROI 时更准确 & $\begin{array}{l}\text { 边界可能出现伪影, 且对 } \\
\text { 计算资源要求高 }\end{array}$ \\
\hline \multirow{8}{*}{$\begin{array}{l}\text { 直接参与压 } \\
\text { 缩的机器 } \\
\text { 学习 }\end{array}$} & {$[25]$} & 2008 & $\begin{array}{l}\text { 使用累计分布函数将像素映射到概率 } \\
\text { 空间 }\end{array}$ & 解决神经网络压缩收玫慢的问题 & 压缩损失较高 \\
\hline & {$[26]$} & 2008 & 使用提升小波方法进行预处理 & 增强图像的局部相似性 & \\
\hline & {$[27]$} & 2011 & 使用 5 层的反向传播网络 & 结构简单 & 没有变换到频域, 受分块 \\
\hline & & & & & 大小的影响大 \\
\hline & {$[28]$} & 2013 & 结合混沌映射与神经网络 & 与一般神经网络相比容错能力更强 & $\begin{array}{l}\text { 理论基础复杂但压缩效 } \\
\text { 果一般 }\end{array}$ \\
\hline & {$[29]$} & 2011 & 利用自编码器隐藏层比输人层神经元 & 较为灵活, 叠加不同层数的自编码器就 & 叠加多层自编码器反向 \\
\hline & $\begin{array}{l}{[30]} \\
{[31]}\end{array}$ & $\begin{array}{l}2011 \\
2018\end{array}$ & 少, 得到输人的低维表示 & 可以得到不同尺度的压缩 & 传播造成的损失会累积 \\
\hline & {$[32]$} & 2019 & 利用 CRNN 作为编码器与解码器 & $\begin{array}{l}\text { 作为独立编码器而非对传统方法的改进, } \\
\text { 潜力更大, 不受传统方法精度限制 }\end{array}$ & $\begin{array}{l}\text { 编码过程的可解释性 } \\
\text { 下降 }\end{array}$ \\
\hline
\end{tabular}

对压缩图像质量常用的度量指标有均方误差 (mean square error, MSE)、峰值信噪比(peak signal-to-noise ratio, PSNR) 和结构相似性 (structural similarity, SSIM $)^{[33]}$. 基于机器学习的医学图像压 缩方法的质量对比如表 3 所示. 其中, 有些方法有 数种设置, 压缩率为 $0.01 \sim 0.90$, 重构图像质量也各 不相同. 由于医学图像对图像质量要求较高, 表 3 仅 统计了使重构图像质量最高的压缩效果.

\section{3 结 语}

目前，基于 VQ 的方法、基于变换编码的方法 和基于分形的方法仍然是主流的医学图像压缩方 法, 机器学习在医学图像上用于这些方法的改进, 它加快了这些传统方法的编码速度, 提高了压缩 率, 增强了重构图像质量, 加强了在不同类型医学 图像数据上应用压缩的泛化性能. 值得注意的 
表 3 基于机器学习的医学图像压缩方法压缩效果统计

\begin{tabular}{|c|c|c|c|c|c|}
\hline 方法分类 & 文献 & 出版年 & 数据 & 压缩率(压缩后大小/原始大小) & 图像质量 \\
\hline \multirow{9}{*}{$\begin{array}{l}\text { 机器学习结 } \\
\text { 合 VQ }\end{array}$} & {$[5]$} & 2009 & 彩色眼底图像 & & $36.75 \sim 42.26 \mathrm{~dB}$ (PSNR) \\
\hline & {$[6]$} & 2012 & 肝脏 $\mathrm{CT}$ 图像/脑部 $\mathrm{CT}$ 图像 & 0.13 (肝脏) $/ 0.13$ (脑部) & $0.94 / 0.95$ (SSIM) \\
\hline & [7] & 2018 & $\begin{array}{l}\text { 一般 X 射线图像、乳腺钼靶 } \\
\text { 图像、腹部 CT 图像等 }\end{array}$ & $\begin{array}{l}0.18 \text { (一般 } X \text { 射线) } / 0.18(\text { 乳 } \\
\text { 腺 }) / 0.18 \text { (腹部) }\end{array}$ & $27.73 / 50.46 / 33.28 \mathrm{~dB}$ (PSNR) \\
\hline & {$[8]$} & 2012 & 乳腺钿靶图像 & & $42.97 \mathrm{~dB}$ (PSNR) \\
\hline & [9] & 2005 & 乳腺钼靶图像 & 0.25 & $35.48 \mathrm{~dB}$ (PSNR) \\
\hline & $\begin{array}{l}{[10]} \\
{[11]}\end{array}$ & $\begin{array}{l}2006 \\
2006\end{array}$ & $\begin{array}{l}\text { 眼底图像、手部 X 射线图像、 } \\
\text { 肺部 X 射线图像等 }\end{array}$ & $\begin{array}{l}0.025 \text { (眼底) } / 0.025 \text { (手部)/ } \\
0.025 \text { (肺部) }\end{array}$ & $\begin{array}{l}30.19 / 31.19 / 30.35 \mathrm{~dB} \text { (PSNR), 但由 } \\
\text { 于重构误差也被传输, 因此总体完 } \\
\text { 全无损 }\end{array}$ \\
\hline & {$[13]$} & 2010 & 膝关节 MRI 图像 & 0.06 & \\
\hline & {$[14]$} & 2009 & 脑部 MRI 图像 & & $38.46 \mathrm{~dB}$ (PSNR) \\
\hline & {$[15]$} & 2006 & 脑部 MRI 图像 & & $32.38 \mathrm{~dB}$ (PSNR) \\
\hline \multirow{4}{*}{$\begin{array}{l}\text { 机器学习结 } \\
\text { 合变换编码 }\end{array}$} & {$[16]$} & 2006 & 脑部 MRI 图像 & & $44.10 \mathrm{~dB}$ (PSNR) \\
\hline & {$[17]$} & 2009 & 一般 X 射线图像 & $0.10 \sim 0.90$ & \\
\hline & {$[18]$} & 2012 & 肌肉 MRI 图像 & 0.78 & $71.79 \mathrm{~dB}$ (PSNR, 近似无损) \\
\hline & {$[19]$} & 2014 & 脑部 MRI 图像/肝脏 $\mathrm{CT}$ 图像 & $0.73 / 0.78$ & \\
\hline \multirow{3}{*}{$\begin{array}{l}\text { 机器学习结 } \\
\text { 合分形 }\end{array}$} & {$[20]$} & 2013 & 脑部 MRI 图像 & 0.22 & $31.60 \mathrm{~dB}$ (PSNR) \\
\hline & {$[21]$} & 2013 & 脑部 MRI 图像 & 0.28 & $39.15 \mathrm{~dB}$ (PSNR) \\
\hline & {$[22]$} & 2016 & 肺部 CT 图像/脑部 MRI 图像 & $\begin{array}{l}\text { 约为 } 0.5 \text { (肺部)/约为 } 0.5 \text { (脑 } \\
\text { 部)(文中未给出精确值) }\end{array}$ & $\begin{array}{l}\text { 约为 } 30 \mathrm{~dB} / \text { 约为 } 25 \mathrm{~dB} \text { (PSNR, 文 } \\
\text { 中未给出精确值) }\end{array}$ \\
\hline \multirow{2}{*}{$\begin{array}{l}\text { 机器学习结 } \\
\text { 合基于 ROI/ } \\
\text { VOI }\end{array}$} & [23] & 2014 & 脑部 MRI 图像 & 约为 0.2 (文中未给出精确值) & $\begin{array}{l}\text { 约为 } 65 \mathrm{~dB} \text { (PSNR, 文中未给出 } \\
\text { 精确值, 近似无损) }\end{array}$ \\
\hline & {$[24]$} & 2018 & 脊椎 MRI 图像 & 0.81 & $60.97 \mathrm{~dB}$ (PSNR, 近似无损) \\
\hline \multirow{8}{*}{$\begin{array}{l}\text { 直接参与压 } \\
\text { 缩的机器 } \\
\text { 学习 }\end{array}$} & {$[25]$} & 2008 & 腹部/胸部 MRI 图像 & 0.25 (腹部) $/ 0.25$ (胸部) & 29.42/29.61 dB (PSNR) \\
\hline & {$[26]$} & 2008 & 胸部 CT 图像/胸部 MRI 图像 & & $31.50 / 30.97 \mathrm{~dB}$ (PSNR) \\
\hline & {$[27]$} & 2011 & 膝关节 MRI 图像 & 0.03 & $39.56 \mathrm{~dB}$ (PSNR) \\
\hline & {$[28]$} & 2013 & $\begin{array}{l}\text { 脑部 MRI 图像/腹部超声图 } \\
\text { 像 }\end{array}$ & 0.50(脑部)/0.50(腹部) & 27.26/30.58 dB (PSNR) \\
\hline & {$[29]$} & 2011 & 乳腺钿靶图像 & 0.50 & $\begin{array}{l}\text { 约为 } 20 \text { (MSE, 文中未给出精确 } \\
\text { 值) }\end{array}$ \\
\hline & {$[30]$} & 2011 & 一般 X 射线图像 & 约为 0.56 (文中未给精确值) & \\
\hline & {$[31]$} & 2018 & 乳腺钼靶图像 & & $\begin{array}{l}\text { 文中未给出精确值, 约为 } 55 \mathrm{~dB} \\
\text { (PSNR), 约为 } 0.95 \text { (SSIM) }\end{array}$ \\
\hline & {$[32]$} & 2019 & 胸部 X 射线图像 & 0.125 & 35.9 dB (PSNR), 0.96 (SSIM) \\
\hline
\end{tabular}

是, 已有越来越多直接使用机器学习的压缩方法, 它们不受传统的压缩方法的上限约束，有很大的 发展潜力。

上述使用机器学习的医学图像压缩方法大多 是有损压缩方法，文献[18,23-24]为近似无损方法， 只有文献[10]由于将重构图像与真实图像的误差 与编码结果一起保存下来, 为完全无损方法. 基于 $\mathrm{VQ}$ 、变换编码和分形的方法本身就是有损压缩方 法, 利用机器学习对其进行改进并不能改变其有 损的事实. 此外, 由于神经网络的黑盒性质, 利用 神经网络直接对图像进行压缩缺乏可解释性和可 逆性, 同样无法完全消除图像损失.
随着数据体量的不断增大, 医学图像的传输 和存储对于有损压缩的容忍度正在逐步增加. 同 时, 基于 ROI 的方法可以在享受有损压缩带来的 高压缩率的同时保留重要区域的特征信息. 从表 2 和表 3 的统计可以看出, 机器学习结合基于 ROI 的方法在平均压缩率和平均重构质量上都达到了 较好的效果, 原因是医学图像的 ROI 较小, 包含信 息较为丰富, 而 ROI 外部的背景区域主要是空腔 或者平滑的组织区域, 包含信息相对稀少. 对 ROI 进行无损压缩、对外部区域进行有损压缩的方式可 以有效地结合有损压缩高压缩率和无损压缩高图 像质量的特点, 从中找到最佳的平衡点, 很可能成 
为基于机器学习的视频压缩方法的主流发展方向.

现有的机器学习用于压缩的方法大多是使用 机器学习分割 ROI，再用传统方法压缩; 但是，随 着机器学习直接压缩图像方法的发展, 分割、压缩 均使用机器学习的方法可能将成为未来最有效的 医学图像压缩方式，给医学影像技术的发展带来 更大的便利

\section{参考文献(References):}

[1] Liu F, Hernandez-Cabronero M, Sanchez V, et al. The current role of image compression standards in medical imaging $[\mathrm{J}]$. Information, 2017, 8(4): Article No.131

[2] Tabuman D S, Marcellin M W, Rabbani M. JPEG2000: image compression fundamentals, standards and practice[J]. Journal of Electronic Imaging, 2002, 11(2): 286-287

[3] Shapiro J M. Embedded image coding using zerotrees of wavelet coefficients[J]. IEEE Transactions on Signal Processing, 1993, 41(12): 3445-3462

[4] Said A, Pearlman W A. A new, fast, and efficient image codec based on set partitioning in hierarchical trees[J]. IEEE Transactions on Circuits and Systems for Video Technology, 1996, 6(3): $243-250$

[5] Setiawan A W, Suksmono A B, Mengko T R. Color medical image vector quantization coding using $k$-means: retinal image[C] //Proceedings of the 13th International Conference on Biomedical Engineering. Heidelberg: Springer, 2009: 911-914

[6] Jiang H Y, Ma Z Y, Hu Y, et al. Medical image compression based on vector quantization with variable block sizes in wavelet domain[J]. Computational Intelligence and Neuroscience, 2012: Article No.541890

[7] Kouanou A T, Tchiotsop D, Tchinda R, et al. A machine learning algorithm for biomedical images compression using orthogonal transforms[J]. International Journal of Image, Graphics and Signal Processing, 2018, 10(11): 38-53

[8] Hsu W Y. Improved watershed transform for tumor segmentation: application to mammogram image compression[J]. Expert Systems with Applications, 2012, 39(4): 3950-3955

[9] Meyer-Bäse A, Jancke K, Wismüller A, et al. Medical image compression using topology-preserving neural networks[J]. Engineering Applications of Artificial Intelligence, 2005, 18(4): 383-392

[10] Ashraf R, Akbar M. Absolutely lossless compression of medical images[C] //Proceedings of the 27th Annual Conference of the IEEE Engineering in Medicine and Biology Society. Los Alamitos: IEEE Computer Society Press, 2006: 4006-4009

[11] Ashraf R, Akbar M. Adaptive architecture neural nets for medical image compression[C] //Proceedings of the IEEE International Conference on Engineering of Intelligent Systems. Los Alamitos: IEEE Computer Society Press, 2006: 1-4

[12] Kohonen T. The self-organizing map[J]. Proceedings of the IEEE, 1990, 78(9): 1464-1480

[13] Guo H, Wang Y X. Wavelet packet and neural network basis medical image compression[C] //Proceedings of the Interna- tional Conference on E-Product E-Service and E-Entertainment. Los Alamitos: IEEE Computer Society Press, 2010: 1-3

[14] Zhao Ming, Xiahou Chunhong. Medical image compression method based on improved self-organizing mapping network[J]. Journal of Clinical Rehabilitative Tissue Engineering Research, 2009, 13(30): 5903-5906(in Chinese) (赵明, 夏侯春洪. 一种改进型自组织映射网络的医学图像 压缩方法 [J]. 中国组织工程研究与临床康复，2009，13(30): 5903-5906)

[15] Karlık B. Medical image compression by using vector quantization neural network (VQNN)[J]. Neural Network World, 2006, 16(4): 341-348

[16] Northan B, Dony R D. Image compression with a multiresolution neural network $[\mathrm{J}]$. Canadian Journal of Electrical and Computer Engineering, 2006, 31(1): 49-58

[17] Khashman A, Dimililer K. Medical radiographs compression using neural networks and Haar wavelet[C] //Proceedings of the IEEE EUROCON. Los Alamitos: IEEE Computer Society Press, 2009: 1448-1453

[18] Vijideva R. Neural network-wavelet based dicom image compression and progressive transmission[J]. International Journal of Engineering Science and Advanced Technology, 2012, 2(4): $702-710$

[19] Saudagar A K J, Syed A S. Image compression approach with ridgelet transformation using modified neuro modeling for biomedical images[J]. Neural Computing and Applications, 2014, 24(7-8): 1725-1734

[20] Bhavani S, Thanushkodi K G. Comparison of fractal coding methods for medical image compression[J]. IET Image Processing, 2013, 7(7): 686-693

[21] Lakshmi G M, Rao S R M. A novel algorithm for image compression based on fractal and neural networks[J]. International Journal of Engineering and Innovative Technology, 2013, 3(4): 8-15

[22] Perumal B, Rajasekaran M P, Prasath T A. Efficient hybrid approach for compression of multi modal medical images[C] //Proceedings of the International Conference on Theoretical Computer Science and Discrete Mathematics. Heidelberg: Springer, 2016: 251-261

[23] Thomas D S, Moorthi M, Muthalagu R. Medical image compression based on automated ROI selection for telemedicine application[J]. International Journal of Engineering and Computer Science, 2014, 3(1): 3638-3642

[24] Fan B. Selective compression of medical images via intelligent segmentation and 3D-SPIHT coding[D]. Milwaukee: University of Wisconsin-Milwaukee. Department of Compter Science, 2018

[25] Durai S A, Saro E A. Image compression with back-propagation neural network using cumulative distribution function[J]. International Journal of Computer and Information Engineering, 2008, 2(5): 1571-1575

[26] Wei Huanhuan. Research of medical image compression technology based on DICOM standards[D]. Jinan: Shandong University, 2008(in Chinese) (魏欢欢. 基于 DICOM 标准的医学图像压缩技术研究[D]. 
济南: 山东大学, 2008)

[27] Yeo W K, Yap D F W, Oh T H, et al. Grayscale medical image compression using feedforward neural networks $[\mathrm{C}]$ //Proceedings of the IEEE International Conference on Computer Applications and Industrial Electronics. Los Alamitos: IEEE Computer Society Press, 2011: 633-638

[28] Zhang Yi. Study on chaotic neural network algorithms and its application in medical image processing[D]. Dalian: Dalian University of Technology, 2013(in Chinese)

(张诣. 混沌神经网络算法研究及其在医学图像处理中的应 用[D]. 大连: 大连理工大学, 2013)

[29] Tan C C, Eswaran C. Using autoencoders for mammogram compression[J]. Journal of Medical Systems, 2011, 35(1): 49-58

[30] Senthilkumaran N, Suguna J. Neural network technique for lossless image compression using X-ray images[J]. International Journal of Computer and Electrical Engineering, 2011, 3(1): 17-23

[31] Kar A, Karri S P K, Ghosh N, et al. Fully convolutional model for variable bit length and lossy high density compression of mammograms[C] //Proceedings of the CVPR Workshops. Los Alamitos: IEEE Computer Society Press, 2018: 2591-2594

[32] Sushmit A S, Zaman S U, Humayun A I, et al. X-ray image compression using convolutional recurrent neural networks[C] //Proceedings of the IEEE EMBS International Conference on Biomedical and Health Informatics. Los Alamitos: IEEE Computer Society Press, 2019: 1-4

[33] Wang Z, Bovik A C, Sheikh H R, et al. Image quality assessment: from error visibility to structural similarity[J]. IEEE Transactions on Image Processing, 2004, 13(4): 600-612 